PROCEEDINGS OF THE

AMERICAN MATHEMATICAL SOCIETY

Volume 135, Number 7, July 2007, Pages 2073-2080

S 0002-9939(07)08751-5

Article electronically published on February 2, 2007

\title{
COLLAPSED INDECOMPOSABLE CONTINUA IN AREA PRESERVING \\ TWO-DIMENSIONAL DYNAMICAL SYSTEMS
}

\author{
JUDY KENNEDY
}

(Communicated by Michael Handel)

\begin{abstract}
While invariant indecomposable continua can occur in two dimensional area preserving dynamical systems, it is often the case that processes that would normally produce these continua instead produce a collapsed version of the continua because of the area preserving constraints. The collapsed continuum and the dynamics on it have a strong relationship to an indecomposable continuum in a related dynamical system. We also prove that the presence of a homoclinic point of a saddle point $p$ in such a system has a branch $W^{u+}(p)$ of its unstable manifold that is inaccessible from the complement of the closure of $W^{u+}(p)$.
\end{abstract}

Homoclinic tangles (the result of the intersection of the unstable and stable manifolds of a saddle fixed point) in area preserving dynamical systems have been the subject of much study in the past forty years or so. Here we investigate these systems on the sphere $S^{2}$ from a new perspective. We prove that they are closely related to dynamical systems on $S^{2}$ that admit invariant indecomposable continua, and that these tangles inherit much of the structure of the indecomposable continuum. Since much about indecomposable continua, especially in a two-dimensional space, is understood, we hope that this will further help in understanding the structure of homoclinic tangles.

In order to understand the space-filling unstable foliation of closed chaotic Hamiltonian systems, physicists recently have been "leaking up" such systems. That is, they cut out a finite (small) region of phase space, the leak, through which escape of trajectories is possible, and have as a consequence discovered that this leads to transient chaotic behavior of nearly all trajectories. (See [STN] and [SHA, for example.) The physicists' evidence is mostly numerical. In this paper, we establish rigorous results, but our strategy is related to that of the physicists. However, rather than "leak up", we add a set to the original space in order to pull apart layers. Our purpose is the same though: we wish to understand more about the structure of orbits of such a system.

All indecomposable continua share certain structure. First, an indecomposable continuum is a continuum (a compact, connected, metric space) with the property

Received by the editors March 3, 2006.

2000 Mathematics Subject Classification. Primary 37C29, 54H20.

Key words and phrases. Homoclinic point, indecomposble continuum, area preserving, two dimensional.

This research was supported by the National Science Foundation.

(C) 2007 American Mathematical Society Reverts to public domain 28 years from publication 
that it cannot be expressed as the union of two proper subcontinua. (More details follow in the next section.) Equivalently, an indecomposable continuum is a continuum that has the property that each of its subcontinua is nowhere dense in the continuum. If $X$ is an indecomposable continuum and $x \in X$, the composant of $x$, denoted $C p s(x)$, is the union of all proper subcontinua of $X$ that contain $x$. Each composant of $X$ is a connected, first category set which is dense in $X$. The collection $C=\{\operatorname{Cps}(x): x \in X\}$ partitions the space into an uncountable collection of sets. Thus, two composants intersect if and only if they are the same set. The composants are like dense highways in the set: each highway is "close" to all other highways, but no two different highways (composants) ever intersect.

Indecomposable continua can occur as invariant sets in area preserving dynamical systems in the plane: Michael Handel $\left[\mathrm{H}\right.$ ] constructed a $C^{\infty}$ area preserving diffeomorphism of the plane with the pseudocircle, a specific example of an indecomposable continuum, as an invariant minimal set. These particular indecomposable continua contain no nontrivial images of arcs, and cannot arise from a homoclinic point associated with a saddle fixed point.

If one has a saddle fixed point $p$ in an area preserving system in the plane such that one branch of the unstable manifold $W^{u+}(p)$ intersects the stable manifold $W^{s}(p)$, but $W^{u+}(p)$ is not contained in $W^{s}(p)$, and $W^{u+}(p)$ is invariant in the system, then $\overline{W^{u+}(p)}$ can be an indecomposable, connected closed set, provided one allows $\overline{W^{u+(p)}}$ to be noncompact. (See $\left[\mathrm{KY}\right.$, for example.) However, if $\overline{W^{u+}(p)}$ is required to be compact, then an indecomposable continuum will not result, because of the area preserving constraint. The area preserving constraint "forces out" any space between layers in the continuum.

The paper heavily uses the results of Marcy Barge [B] about the structure of the closure of one branch of an unstable manifold when it intersects one branch of the stable manifold of a saddle point.

\section{Background, notation, And Definitions}

Let $F: M \rightarrow M$ be a $C^{1}$-diffeomorphism of the $m$-manifold $M$ and let $p \in M$ be a hyperbolic fixed point of $F$ with stable manifold $W^{s}(p)$ and one-dimensional unstable manifold $W^{u}(p)$. Let $W^{u+}(p)$ be one of the branches of $W^{u}(p)$ and assume that $F\left(W^{u+}(p)\right)=W^{u+}(p)$. By Hartman's Theorem there is a homeomorphism $\psi: B_{1} \rightarrow M^{\prime}$, where $M^{\prime}$ is some closed neighborhood of the hyperbolic fixed point $p$,

$$
B_{1}=\left\{\left(x_{1}, x_{2}, \ldots, x_{m}\right) \in \mathbb{R}^{m}: \sum_{i=1}^{m-1} x_{i}^{2} \leq 1,0 \leq x_{m} \leq 1\right\},
$$

and such that

$$
\psi\left(\left\{\left(x_{1}, x_{2}, \ldots, x_{m-1}, 0\right) \in B_{1}: \sum_{i=1}^{m-1} x_{i}^{2} \leq 1\right\}\right)=W_{l o c}^{s}(p),
$$

and

$$
\psi\left(\left\{\left(0,0, \ldots, 0, x_{m}\right) \in B_{1}: 0 \leq x_{m} \leq 1\right\}\right)=W_{l o c}^{u+}(p)
$$

(where $W_{l o c}^{s}(p)$ and $W_{l o c}^{u}(p)$ denote the local stable and unstable manifolds of $p$, respectively), and $A=\psi^{-1} \circ F \circ \psi$ is linear where defined.

We consider the following conditions (Barge's "mild conditions") on $F$ :

A1) $\overline{W^{u+}(p)}$ is compact. 
A2) There is an $\operatorname{arc} \alpha$ in $\psi\left(B_{1}\right) \cap W^{u+}(p)$ such that $\alpha \cap W_{l o c}^{s}(p) \neq \varnothing$ and $\alpha \varsubsetneqq W_{l o c}^{s}(p)$.

A3) There is a closed neighborhood relative to $W_{l o c}^{s}(p), S$, which is contained in $W_{l o c}^{s}(p) \backslash\{p\}$ such that $S \cap W^{u+}(p)=\varnothing$. (If $W^{s}(p)$ is topologically one-dimensional (so $M$ is a two-manifold), then $S$ is an interval in $W_{l o c}^{s}(p)$ which does not intersect $W^{u+}(p)$.)

A continuum is a compact, connected metric space. If $X$ and $Y$ are continua, and $Y \subset X$, then $Y$ is a subcontinuum of $X$. If $Y$ is a subcontinuum of $X$, but $Y \neq X$, then $Y$ is a proper subcontinuum of $X$. A continuum is indecomposable if it is not the union of two proper subcontinua. Equivalently, a continuum is indecomposable if every proper subcontinuum has empty interior (relative to the continuum). More generally, a space is indecomposable if it is connected and not the union of two closed proper connected subsets.

If $x$ is a point in an indecomposable continuum $X$, then the composant of $x$, $\operatorname{Cps}(x)=\bigcup\{A: x \in A$ and $A$ is a proper subcontinuum of $X\}$. If two composants $\operatorname{Cps}(x)$ and $\operatorname{Cps}(y)$ intersect (in an indecomposable continuum), then $\operatorname{Cps}(x)=$ $\operatorname{Cps}(y)$. The collection $\mathcal{C}$ of all composants of an indecomposable continuum has cardinality $c$, the cardinality of the real numbers. Each composant $C p s(x)$ is dense and connected in the indecomposable continuum and is the union of a countable collection of nowhere dense continua.

If $X$ is a continuum, $K$ is a closed subset of $X$, and $A \subset K$, then $A$ is accessible from $X \backslash K$ if there is an $\operatorname{arc} P$ in $X$ such that $P \cap K$ consists of exactly one point and that point is in $A$.

If $X$ is a metric space, we use $d$ to denote the metric on $X$. For $x \in X, \epsilon>0$, $D_{\epsilon}(x)=\{y \in X: d(x, y)<\epsilon\}$.

Theorem 1 (Marcy Barge). Suppose that $F: M \rightarrow M$ is a $C^{1}$ diffeomorphism with hyperbolic fixed point $p$ that satisfies A1)-A3). Then $\overline{W^{u+}(p)}$ is an indecomposable continuum.

The lemma below is contained in Barge's proof of the above theorem. Since we use it, we give its proof here. Note that the lemma does not require that condition A3) above holds.

Lemma 2 (Marcy Barge). Suppose that $F: M \rightarrow M$ is a $C^{1}$ diffeomorphism with hyperbolic fixed point $p$ that satisfies A1)-A2) and that each branch of the unstable manifold maps to itself. Let $\psi: B_{1} \rightarrow M^{\prime}$ denote the homeomorphism from $B_{1}$ onto a neighborhood of the hyperbolic fixed point $p$ guaranteed by Hartman's Theorem. Let $G:[0, \infty) \rightarrow W^{u+}(p)$ be a continuous, one-to-one, onto parametrization of $W^{u+}(p)$. Then $\overline{G([y, \infty))}=\overline{W^{u+}(p)}$ for any $y \in[0, \infty)$.

Proof. Let $\alpha=G([x, z])$, with $G(z) \in W_{\text {loc }}^{s}(p)$, be an arc in $\psi\left(B_{1}\right) \cap W^{u+}(p)$ such that $\alpha \varsubsetneqq W_{l o c}^{s}(p)$. For $n \geq 0$, let $\alpha_{n}$ be the connected component of $F^{n}(\alpha) \cap \psi\left(B_{1}\right)$ containing $F^{n}(G(z))$. Hyperbolicity of $F$ at $p$ implies that $\lim \sup \alpha_{n}=W_{l o c}^{u+}(p)$. 
For all sufficiently large $n, \alpha_{n} \subset G([y, \infty))$ so that $\left.\overline{G([y, \infty)}\right) \supset W_{l o c}^{u+}(p)$. Thus,

$$
\begin{aligned}
\overline{W^{u+}(p)} & =\overline{\bigcup_{n \geq 0} F^{n}\left(W_{l o c}^{u+}(p)\right)} \\
& \subset \overline{\bigcup_{n \geq 0} F^{n}(\overline{G([y, \infty)})}=\overline{\bigcup_{n \geq 0}\left(\overline{F^{n}(G([y, \infty))}\right)} \\
& \subset \overline{G([y, \infty))} .
\end{aligned}
$$

Lemma 3. Suppose $f$ is an area preserving homeomorphism from a compact metric space $X$ to itself. If $U$ is an open set in $X, \widehat{U}:=\bigcup_{i=1}^{\infty} f^{i}(U)$, then $\widehat{U} \cap U$ is dense in $U$, and $f(\widehat{U}) \subset \widehat{U}$ is dense in $\widehat{U}$, and $\mu(\widehat{U} \backslash f(\widehat{U}))=0$. (We assume that the measure function $\mu$ is regular, i.e., each nonempty open set has measure greater than 0.)

Proof. Suppose $O$ is a nonempty open set that is contained in $U$. Then $\widehat{O}:=$ $\bigcup_{i=1}^{\infty} f^{i}(O) \subset \widehat{U}$, and there is at least $n \geq 1$ such that $f^{n}(O) \cap O \neq \varnothing$. (Otherwise $\left\{f^{m}(O): m \geq 0\right\}$ is a pairwise disjoint collection of open sets having the same measure, and that cannot happen.) Then $\widehat{U} \cap U$ is dense in $U$.

Now $f(\widehat{U})=\bigcup_{i=2}^{\infty} f^{i}(U) \subset \widehat{U}=\bigcup_{i=1}^{\infty} f^{i}(U)$. If $y \in \widehat{U}, y \in f^{m}(U)$ for some $m \geq 1$. If $m>1, y \in f(\widehat{U})$. If $m=1$, then an argument similar to that above shows that $f(\widehat{U}) \cap O \neq \varnothing$, where $O$ is any open set containing $y$ and contained in $f(U)$. Since $f(\widehat{U})$ is an open set dense in the open set $\widehat{U}, \mu(\widehat{U} \backslash f(\widehat{U}))=0$.

Theorem 4. Suppose $f$ is an area preserving diffeomorphism of $S^{2}, p$ is a saddle point of $f, f\left(W^{u+}(p)\right)=W^{u+}(p), f\left(W^{s+}(p)\right)=W^{s+}(p)$, and $q \in\left(W^{u+}(p) \cap\right.$ $\left.W^{s+}(p)\right) \backslash\{p\}$, where $W^{u+}(p)$ is one branch of the unstable manifold of $p$ and $W^{s+}(p)$ is one branch of the stable manifold of $p$. Then $\overline{W^{u+}(p)}=\overline{W^{s+}(p)}$.

Proof. We may assume that $W^{s+}(p) \neq W^{u+}(p)$, for if this is the case, the theorem is true. There is a continuous, one-to-one, onto function $u:[0, \infty) \rightarrow W^{u+}(p)$ such that $u(0)=p$, and a continuous, one-to-one, onto function $s:[0, \infty) \rightarrow W^{s+}(p)$ such that $s(0)=p$. For an interval $[a, b] \subset[0, \infty)$, let $[s(a), s(b)]=\{s(t): a \leq t \leq b\}$ and let $[u(a), u(b)]=\{u(t): a \leq t \leq b\}$. Also, let $(s(a), s(b))=\{s(t): a<t<b\}$ and let $(u(a), u(b))=\{u(t): a<t<b\}$.

Suppose that $\overline{W^{u+}(p)}$ does not contain $\overline{W^{s+}(p)}$. Then there is an interval $[\alpha, \beta] \subset[0, \infty)$ such that $[s(\alpha), s(\beta)] \cap \overline{W^{u+}(p)}=\varnothing$. Let $P=[s(\alpha), s(\beta)]$. Since $f\left(W^{u+}(p)\right)=W^{u+}(p), f^{n}(P) \cap \overline{W^{u+}(p)}=\varnothing$ for each $n$. There is a point $q \in\left(W^{u+}(p) \cap W^{s+}(p)\right) \backslash\{p\}$, and for some $t_{0}, q=s\left(t_{0}\right)$. There is a positive integer $N_{1}$ such that $f^{N_{1}}(P) \subset Q:=\left[s(0), s\left(t_{0}\right)\right]$.

Without loss of generality, suppose $P \subset Q$. Then the sequence $q, f(q), f^{2}(q), \ldots$ converges to $p$ and is contained in $Q \cap W^{u+}(p)$. For each nonnegative integer $n$, there is $t_{n} \in[0, \infty)$ such that $s\left(t_{n}\right)=q_{n}:=f^{n}(q)$. It must be the case that $t_{0}, t_{1}, \ldots$ is a decreasing sequence converging to 0 . Let $Q_{n}=\left[s\left(t_{n+1}\right), s\left(t_{n}\right)\right]$. For some $n$, $P \subset Q_{n}$. Thus, $q_{n}$ and $q_{n+1}$ are endpoints of the subarc $Q_{n}$ of $W^{s+}(p)$, but they are also endpoints of a subarc $T:=\left[u\left(r_{n}\right), u\left(r_{n+1}\right)\right]$ of $W^{u+}(p)$, where $u\left(r_{n}\right)=q_{n}$, $u\left(r_{n+1}\right)=q_{n+1}$, and $r_{n}<r_{n+1}$. Now it is possible that $T$ intersects $Q_{n}$ at points other than the endpoints of both arcs, but $T \cap P=\varnothing$, since $\overline{W^{u+}(p)} \cap P=\varnothing$. Hence, there is some $t^{*}$ such that $s\left(t^{*}\right) \in T \cap Q$ and $\left(s(\beta), s\left(t^{*}\right)\right) \cap T=\varnothing$. Likewise, there is some $\hat{t}$ such that $s(\hat{t}) \in T \cap Q$ and $(s(\hat{t}), s(\alpha)) \cap T=\varnothing$. Let $\hat{P}=\left[s(\hat{t}), s\left(t^{*}\right)\right] \supset P$. 
Next, $s\left(t^{*}\right)=u\left(r^{*}\right)$ and $s(\hat{t})=u(\hat{r})$ for some $r^{*}$ and $\hat{r}$ in $[0, \infty)$, and if $\hat{T}$ denotes the subarc of $T$ with endpoints $u\left(r^{*}\right)$ and $u(\hat{r})$, then $\hat{P} \cup \hat{T}$ is a simple closed curve containing $P$. Now $S^{2} \backslash(\hat{P} \cup \hat{T})$ has two components. We call the component of $S^{2} \backslash(\hat{P} \cup \hat{T})$ containing $p, C_{\text {out }}$, and the other component $C_{\text {in }}$.

There is a connected open set $R \subset C_{i n}$ such that $P \subset \partial R$, and $R \cap \overline{W^{u+}(p)}=\varnothing$. Suppose $E$ denotes the component of $C_{i n} \backslash \overline{W^{u+}(p)}$ that contains $R$. Then $E$ is open and $\partial E \subset \overline{W^{u+}(p)} \cup \hat{P}$. For $m>1, f^{m}(\hat{P}) \cap \hat{P}=\varnothing$, and for $m=1, f^{1}(\hat{P}) \cap \hat{P}$ can only possibly contain one point, namely $q_{n+1}$, since $\hat{P}$ is "between" $q_{n}$ and $q_{n+1}$. We may also assume (because of Hartman's Theorem) that $f^{m}(\hat{P}) \cap C_{i n}=\varnothing$ for $m>0$. There is $k>0$ such that $f^{k}(E) \cap E \neq \varnothing$, and this implies that $f^{k}(E) \cap \hat{P} \neq \varnothing\left(\right.$ since $f^{m}(E) \cap \overline{W^{u+}(p)}=\varnothing$ for each $\left.m\right)$. However, $f^{k}(\hat{P}) \cap \overline{C_{i n}}=\varnothing$, and this means that $f^{k}(E) \backslash \overline{C_{i n}}$ is a nonempty open set, and $\mu\left(f^{k}(E) \backslash \overline{C_{i n}}\right)>0$. Thus, $f^{k}(E) \cap E$ cannot be dense in $E$, for if it were, then $\mu\left(E \backslash f^{k}(E)\right)=0$ and $\mu(E)=\mu\left(f^{k}(E) \cap E\right)<\mu\left(f^{k}(E)\right)$.

Hence, there is an open set $O$ in $E$ such that $O \cap \overline{f^{k}(E)}=\varnothing$. However, there is an $\operatorname{arc} A$ contained in $E$ which intersects both $O$ and $f^{k}(E)$, so $\partial f^{k}(E) \cap A \neq \varnothing$, which in turn implies that $\overline{W^{u+}(p)} \cap E \neq \varnothing$, since $\partial f^{k}(E) \subset \overline{W^{u+}(p)} \cup f^{k}(\hat{P})$. This is a contradiction. Then $W^{u+}(p)$ is dense in $\overline{W^{s+}(p)}$. Thus, $\overline{W^{s+}(p)} \subset \overline{W^{u+}(p)}$. A similar argument shows that $\overline{W^{u+}(p)} \subset \overline{W^{s+}(p)}$. Thus, the theorem holds.

Theorem 5. Suppose $f$ is an area preserving diffeomorphism of $S^{2}, p$ is a saddle point of $f, f\left(W^{u+}(p)\right)=W^{u+}(p), f\left(W^{s+}(p)\right)=W^{s+}(p), W^{u+}(p) \neq W^{s+}(p)$, and $\left(W^{u+}(p) \cap W^{s+}(p)\right) \backslash\{p\} \neq \varnothing$, where $W^{u+}(p)$ is one branch of the unstable manifold of $p$ and $W^{s+}(p)$ is one branch of the stable manifold of $p$. Then $W^{u+}(p) \backslash\{p\}$ is not accessible from $S^{2} \backslash \overline{W^{u+}(p)}$.

Proof. Suppose $W^{u+}(p) \backslash\{p\}$ is accessible from $S^{2} \backslash \overline{W^{u+}(p)}$. Then there is an arc $A$ such that one endpoint $a$ of $A$ is contained in $W^{u+}(p)$ and $A \backslash\{a\} \subset S^{2} \backslash \overline{W^{u+}(p)}$. Without loss of generality we may assume that $f(A) \cap A=\varnothing$. There is a connected open set $U$ that contains $A \backslash\{a\}$ but $U \cap \overline{W^{u+}(p)}=\varnothing$, and $f(U) \cap U=\varnothing$. However, there is some least $N$ such that $f^{N}(U) \cap U \neq \varnothing$. Then $f^{N}(U) \cup U$ is a connected and locally connected open set such that $\left(U \cup f^{N}(U)\right) \cap \overline{W^{u+}(p)}=\varnothing$. Hence there is an arc $B$ such that $B \subset U \cup f^{N}(U) \cup\left\{a, f^{N}(a)\right\}$ and $B \cap \overline{W^{u+}(p)}=\left\{a, f^{N}(a)\right\}$. (If $A \cap f^{N}(A) \neq \varnothing$, then $A \cap f^{N}(A) \subset U \cup f^{N}(U)$, and $A \cup f^{N}(A)$ contains such an arc. If $A \cap f^{N}(A)=\varnothing$, then let $d$ denote the other endpoint of $A$. Then $d$ and $f^{N}(d)$ are contained in $U \cup f^{N}(U)$, and there is an arc $E$ in $U \cup f^{N}(U)$ with endpoints $d$ and $f^{N}(d)$. Thus, $A \cup E \cup f^{N}(A)$ contains such an arc.) If $P$ denotes the arc in $W^{u+}(p)$ with endpoints $a$ and $f^{N}(a)$, then $B \cup P$ is a simple closed curve. Then $S^{2} \backslash(B \cup P)$ consists of two components, $C_{\text {in }}$ and $C_{\text {out }}$, such that $C_{\text {out }}$ is the component of $S^{2} \backslash(B \cup P)$ that contains $W^{u+}(p) \backslash P$. Then $C_{i n}$ does not intersect $W^{u+}(p)$, but $\partial C_{i n}=B \cup P$.

Now consider $O:=\bigcup_{n \in \mathbb{Z}} f^{n}\left(C_{i n}\right)$. The set $O$ is open and connected, $O$ does not intersect $W^{u+}(p)$, and $W^{u+}(p) \subset \partial O$.

Suppose that $O$ does not intersect $W^{s+}(p)$. There is a continuous, one-to-one, onto function $u:[0, \infty) \rightarrow W^{u+}(p)$ such that $u(0)=p$, and a continuous, one-toone, onto function $s:[0, \infty) \rightarrow W^{s+}(p)$ such that $s(0)=p$. For an interval $[a, b] \subset$ $[0, \infty)$, let $[s(a), s(b)]=\{s(t): a \leq t \leq b\}$ and let $[u(a), u(b)]=\{u(t): a \leq t \leq b\}$. Since $\left(W^{u+}(p) \cap W^{s+}(p)\right) \backslash\{p\} \neq \varnothing$, there is a point $q$ in $\left(W^{u+}(p) \cap W^{s+}(p)\right) \backslash\{p\}$, 
and for some $t_{0}$ and $t_{1}, q=u\left(t_{0}\right)=s\left(t_{1}\right)$. Since $W^{u+}(p) \neq W^{s+}(p), u\left(\left[0, t_{0}\right]\right) \neq$ $s\left(\left[0, t_{1}\right]\right)$. In fact, $u\left(\left[0, t_{0}\right]\right) \varsubsetneqq s\left(\left[0, t_{1}\right]\right)$ and $u\left(\left[0, t_{0}\right]\right) \nsupseteq s\left(\left[0, t_{1}\right]\right)$ (for either would mean that $\left.W^{u+}(p)=W^{s+}(p)\right)$, so there is some point $w \in u\left(\left[0, t_{0}\right]\right) \backslash s\left(\left[0, t_{1}\right]\right)$. Hence there is an $\operatorname{arc} T$ contained in $u\left(\left[0, t_{0}\right]\right)$ such that $T \cap s\left(\left[0, t_{1}\right]\right)=\varnothing$. Using an argument similar to one in the proof of Theorem 4 , there is a simple closed curve $C=\widehat{T} \cup Q \subset u\left(\left[0, t_{0}\right]\right) \cup s\left(\left[0, t_{1}\right]\right)$, such that $T \subset \widehat{T} \subset u\left(\left[0, t_{0}\right]\right)$ (with $T$ and $\widehat{T}$ being arcs), and $Q \subset s\left(\left[0, t_{1}\right]\right)$ with $Q$ being an arc. If $p \notin C$, then $S^{2} \backslash C$ consists of two components, with $C_{\text {out }}^{\prime}$ denoting that component that contains $p$ and $C_{i n}^{\prime}$ denoting the other component. Since $\bar{O} \supset W^{u+}(p)$ but $O \cap W^{s+}(p)=\varnothing, O \subset C_{\text {out }}^{\prime}$. There is a positive integer $M$ such that $f^{M}(Q) \cap Q=\varnothing$ and $f^{M}(\widehat{T}) \cap \widehat{T}=\varnothing$, but $f^{M}\left(C_{i n}^{\prime}\right) \cap C_{i n}^{\prime} \neq \varnothing$. Note that $\partial f^{M}\left(C_{i n}^{\prime}\right)=f^{M}(Q) \cup f^{M}(\widehat{T}) \subset W^{s+}(p) \cup W^{u+}(p)$. Then $f^{M}(\widehat{T}) \cap Q \neq \varnothing$, and it follows that $O \cap C_{i n}^{\prime} \neq \varnothing$, which is a contradiction.

If $p \in C$, then let $C_{\text {in }}^{\prime}$ and $C_{\text {out }}^{\prime}$ denote the components of $S^{2} \backslash C$. For each positive integer $m, f^{m}(Q) \subsetneq Q$, and in order for the measure preserving requirement to hold, $f^{m}(\widehat{T})$ must intersect $Q$ and $C_{\text {out }}^{\prime}$ as well as contain $\widehat{T}$. Then, again, $O$ must intersect $W^{s+}(p)$.

Then there is an $\operatorname{arc} R$ contained in $O \cap W^{s+}(p)$, and $R$ may be chosen so that $R \cap \overline{W^{u+}(p)}=\varnothing$. This contradicts the previous theorem, so our assumption that $W^{u+}(p) \backslash\{p\}$ is accessible from $S^{2} \backslash \overline{W^{u+}(p)}$ must be false.

Theorem 6. Suppose $f$ is an area preserving diffeomorphism of $S^{2}, p$ is a saddle point of $f, f\left(W^{u+}(p)\right)=W^{u+}(p), f\left(W^{s+}(p)\right)=W^{s+}(p), W^{u+}(p) \neq W^{s+}(p)$, and $\left(W^{u+}(p) \cap W^{s+}(p)\right) \backslash\{p\} \neq \varnothing$, where $W^{u+}(p)$ is one branch of the unstable manifold of $p$ and $W^{s+}(p)$ is one branch of the stable manifold of $p$. There is an indecomposable continuum $K$ in $S^{2}$, a homeomorphism $F: S^{2} \rightarrow S^{2}$, and a surjective mapping $h: S^{2} \rightarrow S^{2}$ such that the following hold:

1. F factors over $f$ via the mapping $h$, i.e., $h \circ F=f \circ h$;

2. $F(K)=K$;

3. $h \mid\left(S^{2} \backslash h^{-1}\left(W^{u+}(p) \backslash\{p\}\right)\right)$ is one-to-one;

4. for each point $x$ of $W^{u+}(p) \backslash\{p\}, h^{-1}(x)$ is a copy of an interval; and

5. $h(K)=\overline{W^{u+}(p)}$.

Proof. There is a continuous, one-to-one, onto function $u:[0, \infty) \rightarrow W^{u+}(p)$ such that $u(0)=p$, and a continuous, one-to-one, onto function $s:[0, \infty) \rightarrow W^{s+}(p)$ such that $s(0)=p$. For an interval $[a, b] \subset[0, \infty)$, let $[u(a), u(b)]=\{u(t): a \leq$ $t \leq b\}$ and let $[s(a), s(b)]=\{s(t): a \leq t \leq b\}$. Since for each $a \in[0, \infty)$, $[u(0), u(a)]=[p, u(a)]$ is a differentiable arc in $S^{2}$, we can make the following construction. We make a new copy of $S^{2}$, call it $\widehat{S}^{2}$ to avoid confusion, as follows: for each $a>0$, we carefully replace the point $u(a)$ with an $\operatorname{arc} P(a)$ so that the collection $\mathcal{P}=\{P(a): a \in(0, \infty)\}$ is a continuous collection relative to the Hausdorff topology on closed subsets of $\widehat{S}^{2}$; and we do this so that in the Hausdorff topology, $\lim _{a \rightarrow 0} P(a)=\{p\}$, and $\lim _{a \rightarrow \infty} \operatorname{diam}(P(a))=0$ (where $\operatorname{diam}(P(a)$ ) denotes the diameter of $P(a)$ in $\widehat{S}^{2}$, and the new space is homeomorphic to $S^{2}$. Then $\widehat{S}^{2}=\left\{x: x \in S^{2} \backslash\left(W^{u+}(p) \backslash\{p\}\right)\right\} \cup\{x: x \in P(a)$ for some $a>0\}$.

Note that $S^{2}$ is an upper semicontinuous decomposition of $\widehat{S}^{2}$ obtained by identifying each arc $P(a)$ in $\widehat{S}^{2}$ with the point $u(a)$ in $S^{2}$. That is, we can define $h: \widehat{S}^{2} \rightarrow S^{2}$ by $h(x)=x$ for $x \in S^{2} \backslash\left(W^{u+}(p) \backslash\{p\}\right)$ and for $x \in P(a)$, 
$a>0, h(x)=u(a)$. Now there is a natural extension of $f$ on $S^{2}$ to a homeomorphism $F$ on $\widehat{S}^{2}$. For $x \in S^{2} \backslash\left(W^{u+}(p) \backslash\{p\}\right)$, we define $F(x)=f(x)$. For each $P(a)$, define $F \mid P(a)$ so that $F \mid P(a)$ is a homeomorphism that takes $P(a)$ onto $h^{-1}(f(u(a)))=P(f(u(a)))$. (This can be done so that the resulting map $F: \widehat{S}^{2} \rightarrow \widehat{S}^{2}$ is a homeomorphism.) Furthermore, there are two continuous one-toone maps $\widehat{u}:[0, \infty) \rightarrow \widehat{S}^{2}$ and $\widetilde{u}:[0, \infty) \rightarrow \widehat{S}^{2}$ such that $\widehat{u}(a)$ is one endpoint of $P(a)$ and $\widetilde{u}(a)$ is the other endpoint of $P(a)$ for each $a \in(0, \infty)$. We refer to $\widehat{u}(a)$ as the "left" endpoint of $P(a)$ and $\widetilde{u}(a)$ as the "right" endpoint of $P(a)$.

Note that $h \circ F=f \circ h$. For each $a>0$, let $B(a)$ denote $P(a) \backslash\{\widetilde{u}(a), \widehat{u}(a)\}$. Then $\bigcup_{a>0} B(a)$ is a connected, open set in $\widehat{S}^{2}$. Let $K=\left(h^{-1}\left(\overline{W^{u+}(p)}\right)\right) \backslash\left(\bigcup_{a>0} B_{a}\right)$. Then $F(K)=K$, and $h(K)=\overline{W^{u+}(p)}$. Also, $h \mid K$ is one-to-one except at the points $\widetilde{u}(a), \widehat{u}(a)$ for $a>0$, and $h(\widetilde{u}(a))=h(\widehat{u}(a))=u(a)$. Thus,

$$
h \mid\left(K \cap h^{-1}\left(W^{u+}(p) \backslash\{p\}\right)\right): K \cap h^{-1}\left(W^{u+}(p) \backslash\{p\}\right) \rightarrow\left(W^{u+}(p) \backslash\{p\}\right)
$$

is two-to-one.

Finally, $K$ is an indecomposable continuum by an argument quite similar to that for Barge's Theorem: First, suppose $t>0$, and $z \in K$. There is a sequence $t_{1}, t_{2}, \ldots$ of positive numbers increasing without bound such that $\lim _{n \rightarrow \infty} u\left(t_{n}\right)=$ $h(z)$. Then, with respect to the Hausdorff topology, $\lim _{n \rightarrow \infty} P\left(t_{n}\right)=\{z\}$ (because $\left.\lim _{n \rightarrow \infty} \operatorname{diam} P\left(t_{n}\right)=0\right)$. Then $\overline{\widehat{u}([t, \infty))}=K$. Likewise, $\overline{\widetilde{u}([t, \infty))}=K$. Thus, both $\widehat{u}([t, \infty))$ and $\widetilde{u}([t, \infty))$ are dense in $K$.

Now suppose $K$ is decomposable. Let $H \subset K$ be a proper subcontinuum such that $\operatorname{int}_{K}(H)$ is nonempty. Then $H \cap \widehat{u}([t, \infty)) \neq \varnothing$. However, for $0 \leq t_{1} \leq t_{2}$, $\widehat{u}\left(\left[t_{1}, t_{2}\right]\right)$ has an empty interior in $K$. There are $\epsilon>0$ and points $y_{1}$ and $y_{2}$ in $\widehat{S}^{2}$ such that $y_{1} \in H, y_{2} \in \overline{\widehat{S}^{2} \backslash H}$, and $D_{\epsilon}\left(y_{1}\right) \cap K \subset \operatorname{int}_{K}(H), D_{\epsilon}\left(y_{2}\right) \subset \overline{\widehat{S}^{2} \backslash H}$. Then there are numbers $0 \leq s_{1}<s_{2}<s_{3}$ such that $\widehat{u}\left(s_{1}\right)$ and $\widehat{u}\left(s_{3}\right)$ are in $D_{\epsilon / 4}\left(y_{2}\right)$, $\widehat{u}\left(s_{2}\right)$ is in $K \cap D_{\epsilon / 4}\left(y_{1}\right) \subset \operatorname{int}_{K}(H)$, and diam $P(a)<\epsilon / 4$ for $s_{1} \leq a \leq s_{3}$. Since $\widehat{u}\left(s_{2}\right) \in D_{\epsilon / 4}\left(y_{1}\right)$ and diam $P\left(s_{2}\right)<\epsilon / 4, \widetilde{u}\left(s_{2}\right) \in D_{\epsilon}\left(y_{1}\right) \cap K \subset \operatorname{int}_{K}(H)$. But this gives us a contradiction, for now $\bigcup_{s_{1}<t<s_{3}} P(t)$ is an open connected set in $\widehat{S}^{2}$ which does not intersect $H$, but which separates $H$ into at least two nonempty components. Then no such $H$ exists, and $K$ is indecomposable.

\section{Conclusions}

We can now conclude that a "homoclinic tangle" retains almost all the curve structure of the indecomposable continuum that factors over it. Specifically, a copy of each composant, except for the one that maps to $W^{u+}(p)$, is present in the closure $\overline{W^{u+}(p)}$ of one branch of the unstable manifold $W^{u+}(p)$. If $C$ denotes the composant of $K$ that maps onto $W^{u+}(p), h \mid C$ is two-to-one except at $p$ itself. Furthermore, $h \mid\left(K \backslash h^{-1}\left(W^{u+}(p)\right)\right)$ is one-to-one, and $K \backslash h^{-1}\left(W^{u+}(p)\right)$ is a residual set (actually, a dense $G_{\delta}$-set) in $K$. Much as one can turn the Cantor middlethirds set into an interval by identifying adjacent endpoints (points of the form $\frac{m}{3^{n}}$ ), we can turn a rather special invariant indecomposable continuum in a dynamical system involving a homeomorphism on the sphere into the closure of a branch of the unstable manifold of a saddle point in an area preserving dynamical system on the sphere. Furthermore, under the conditions required in the paper, $W^{u+}(p)$ is not accessible from $S^{2} \backslash \overline{W^{u+}(p)}$. 
One would hope that some version of the results would hold for higher-dimensional manifolds, but this will require different arguments due to the different topology.

\section{REFERENCES}

[B] M. Barge, Homoclinic intersections and indecomposability, Proc. AMS 101 (1987) 541-544. MR0908665 (88k:58119)

[H] M. Handel, A pathological area preserving $C^{\infty}$ diffeomorphism of the plane, Proc. AMS 86 (1982) 163-168. MR0663889 (84f:58040)

[KY] J. Kennedy and J. Yorke, The topology of stirred fluids, Topology and Applications 80 (1997) 201-238. MR 1473918 (99b:54047)

[SHA] M. Sanjuan, T. Horita, and K. Kazuyuki, Opening a closed Hamiltonian map, Chaos 13 (2003) 17-24. MR1964963 (2004a:37083)

[STN] J. Schneider, T. Tél, and Z. Neufeld, Dynamics of "leaking" Hamiltonian systems, Physical Review E 66 (2002). MR1953946 (2003m:82056)

Department of Mathematical Sciences, University of Delaware, Newark, Delaware 19716

E-mail address: jkennedy@math.udel.edu 